\title{
Chinese expert consensus on technical specifications of electromagnetic navigation bronchoscopy in diagnosing peripheral pulmonary lesions
}

\author{
Fangfang Xie ${ }^{1,2 \#}$, Huaping Yang ${ }^{3 \#}$, Rui Huang ${ }^{4 \#}$, Xiaoxuan Zheng ${ }^{1,2}$, Liming Cao ${ }^{3}$, Jingjing Liu ${ }^{3}$, \\ Shuoyao $\mathrm{Qu}^{5}$, Yan Zhang ${ }^{5}$, Shiman $\mathrm{Wu}^{6}$, Jian Zhang ${ }^{5}$, Mingyao $\mathrm{Ke}^{4}$, Jiayuan Sun ${ }^{1,2}$; on behalf of the \\ Interventional \& Minimally Invasive Respiratory Committee of the China Medical Education Association \\ and Expert Assistance Group of the Domestic Electromagnetic Navigation Bronchoscopy Technology \\ ${ }^{1}$ Department of Respiratory Endoscopy, Department of Respiratory and Critical Care Medicine, Shanghai Chest Hospital, Shanghai Jiao Tong \\ University, Shanghai, China; ${ }^{2}$ Shanghai Engineering Research Center of Respiratory Endoscopy, Shanghai, China; ${ }^{3}$ Department of Respiratory \\ Medicine (Department of Respiratory and Critical Care Medicine), National Key Clinical Specialty, Xiangya Hospital, Central South University, \\ Changsha, China; ${ }^{4}$ Department of Respiratory Centre, The Second Affiliated Hospital of Xiamen Medical College, Xiamen, China; ${ }^{5}$ Department of \\ Pulmonary and Critical Care Medicine, Xijing Hospital, Fourth Military Medical University, Xi'an, China; ${ }^{6}$ Department of Respiratory and Critical \\ Care Medicine, First Hospital of Shanxi Medical University, Taiyuan, China \\ \#These authors contributed equally to this work. \\ Correspondence to: Jiayuan Sun. Department of Respiratory Endoscopy, Department of Respiratory and Critical Care Medicine, Shanghai \\ Chest Hospital, Shanghai Jiao Tong University, 241 West Huaihai Road, Shanghai 200030, China. Email: xkyyjysun@163.com; Mingyao Ke. \\ Department of Respiratory Centre, The Second Affiliated Hospital of Xiamen Medical College, 566 Shengguang Road, Xiamen 361000, China. \\ Email: 13860166866@126.com; Jian Zhang. Department of Pulmonary and Critical Care Medicine, Xijing Hospital, Fourth Military Medical \\ University, 127 Changle West Road, Xi'an 710032, China. Email: 13991802890@163.com.
}

Submitted Mar 05, 2021. Accepted for publication Apr 06, 2021.

doi: 10.21037/jtd-21-369

View this article at: http://dx.doi.org/10.21037/jtd-21-369

\section{Introduction}

Electromagnetic navigation bronchoscopy (ENB) is a technology that allows real-time navigation to the target peripheral pulmonary lesions (PPLs) during bronchoscopy. The system uses virtual bronchoscopy and threedimensional (3D) computed tomography (CT) images, in combination with a sensor and electromagnetic field, to enable the navigation of dedicated tools to the target area of the lung. ENB has been used in clinical practice since 2005 but increasingly more in recent years $(1,2)$. Previous studies demonstrated that ENB was effective and safe for the diagnosis of PPLs. Two meta-analyses reported that the sensitivity for diagnosing a PPL and the risk of complications were $71.1 \%$ and $4.2 \%$, and $77 \%$ and $4.4 \%$, respectively $(3,4)$. ENB-guided transbronchial lung biopsy (TBLB) is an important part of such guided bronchoscopy (5).

The superDimension ${ }^{\mathrm{TM}}$ navigation system (Medtronic;
Dublin, Ireland) was a commonly used ENB system in clinics across the United States. It requires a large working channel of the bronchoscope due to the comparably large locatable guide and extended working channel (6). The more recently developed SPiN Navigation System ${ }^{\circledR}\left(\operatorname{Veran}^{\circledR}\right.$ Medical Technologies; St. Louis, MO, USA) combines ENB and electromagnetic navigation-guided transthoracic needle aspiration during the procedure. The tip of its sampling tool carries a sensor that allows the precise tracking of both the position and orientation of the tip in real-time through the electromagnetic field. Finally, the latest ENB system (LungCare navigation system; LungCare Medical Technologies Ltd., Inc, Suzhou, China) can be used in combination with a thin or ultrathin bronchoscope because it has different types of locatable wires with a promising performance $(7,8)$. It has been approved for use in clinical practice since 2016 in China. However, the procedures of this novel technique in diagnosing PPLs are not standardized. This consensus statement has been 
organized and written in a collaborative effort by the Interventional \& Minimally Invasive Respiratory Committee of the Cbina Medical Education Association and the Expert Assistance Group of the Domestic Electromagnetic Navigation Bronchoscopy Technology and aims to develop practice guidelines for the use of this novel ENB system in the diagnosis of PPLs, standardize the procedures, and ensure optimal performance of ENB. All authors have abundant clinical experience in TBLB and ENB technology.

Due to the lack of evidence on the clinical use and diagnostic outcomes of the novel ENB system in the literature, this consensus represents an expert group agreement on general suggestions without formal individual rating of the level of evidence and strength of recommendations. The consensus was reached through discussion and limited to expert suggestions.

\section{Indications and contraindications of ENB}

\section{Indications}

Candidates for ENB diagnosis are patients with (I) PPLs either suspicious for malignancy or deemed benign requiring tissue samples or microbiological evidence for their definitive diagnosis and treatment, and (II) a PPL diameter of more than $8 \mathrm{~mm}$ (9). Patients meeting the above criteria may undergo ENB-guided TBLB, particularly when they have PPLs located in the peripheral third of the lung or the upper lobes, showing a positive bronchus sign, a diameter $\leq 20 \mathrm{~mm}$ (10).

PPLs are defined as lesions beyond the segmental bronchi that are not visible using bronchoscopy (no endobronchial lesion, extrinsic compression, submucosal tumor, and no narrowing, inflammation, or bleeding of the bronchus) (11). The American College of Chest Physicians (ACCP) guidelines recommend ENB in patients with PPLs that are difficult to reach with conventional bronchoscopy (12). The US National Comprehensive Cancer Network (NCCN) guidelines also point out that patients with peripheral nodules may benefit from navigational bronchoscopy (13).

Some diffuse PPLs, such as those seen in interstitial lung disease, are rare indications for ENB-guided diagnostic TBLB but can be considered if targeted sampling is required.

\section{Contraindications}

ENB is a safer technology compared with transthoracic needle aspiration. The most relevant complications result from TBLB, not the use of ENB. Therefore, the primary contraindications are the same as those for TBLB and conventional bronchoscopy (14), including the following: (I) acute myocardial infarction within the last four weeks; (II) active hemoptysis; (III) platelet count of less than $60 \times 10^{9} / \mathrm{L}$ or uncorrected coagulopathy; (IV) unstable hemodynamics; (V) severe respiratory failure $\left(\mathrm{PaO}_{2}\right.$ below $\left.60 \mathrm{mmHg}\right)$; (VI) lesions suspicious of pulmonary arteriovenous fistula, aneurysm, hemangioma, or cysticercosis; (VII) other cardiovascular risk factors (malignant arrhythmia, unstable angina pectoris, hypertensive crisis, severe pulmonary hypertension, intracranial hypertension, acute cerebrovascular events, aortic dissection, aortic aneurysm); (VIII) pregnancy; (IX) severe mental illness; and (X) general weakness or other conditions that are not compatible with TBLB.

\section{Potential audiences of this consensus statement}

This consensus statement is aimed at clinical staff engaged in the bronchoscopic diagnosis and treatment of PPLs at departments of respiratory medicine, endoscopy, oncology, thoracic surgery, and related disciplines, as well as teaching and research staff engaged in bronchoscopy.

\section{Primary devices and components}

\section{Compatible bronchoscopes}

The novel LungCare ENB system can be used in combination with almost all types of bronchoscopes, including ultrathin, thin, standard, and therapeutic bronchoscopes from Olympus, Pentax, and Fuji, and the single-use scope from Ambu. Olympus bronchoscopes with outer diameters of $5.9 \mathrm{~mm}, 4.8$ or $4.9 \mathrm{~mm}$, and 4.0 or $4.2 \mathrm{~mm}$ are considered as therapeutic, standard, and thin bronchoscopes, respectively (15). An outer diameter $\leq 3.5 \mathrm{~mm}$ is defined as ultrathin. Bronchoscopes from other brands are similar.

\section{The novel ENB system}

The LungCare ENB system consists of a workstation with computer software, an electromagnetic location board, a locatable wire, and a position detection wire (Figure 1).

The computer software enables detailed procedure planning and creates a $3 \mathrm{D}$ view and virtual image of the 


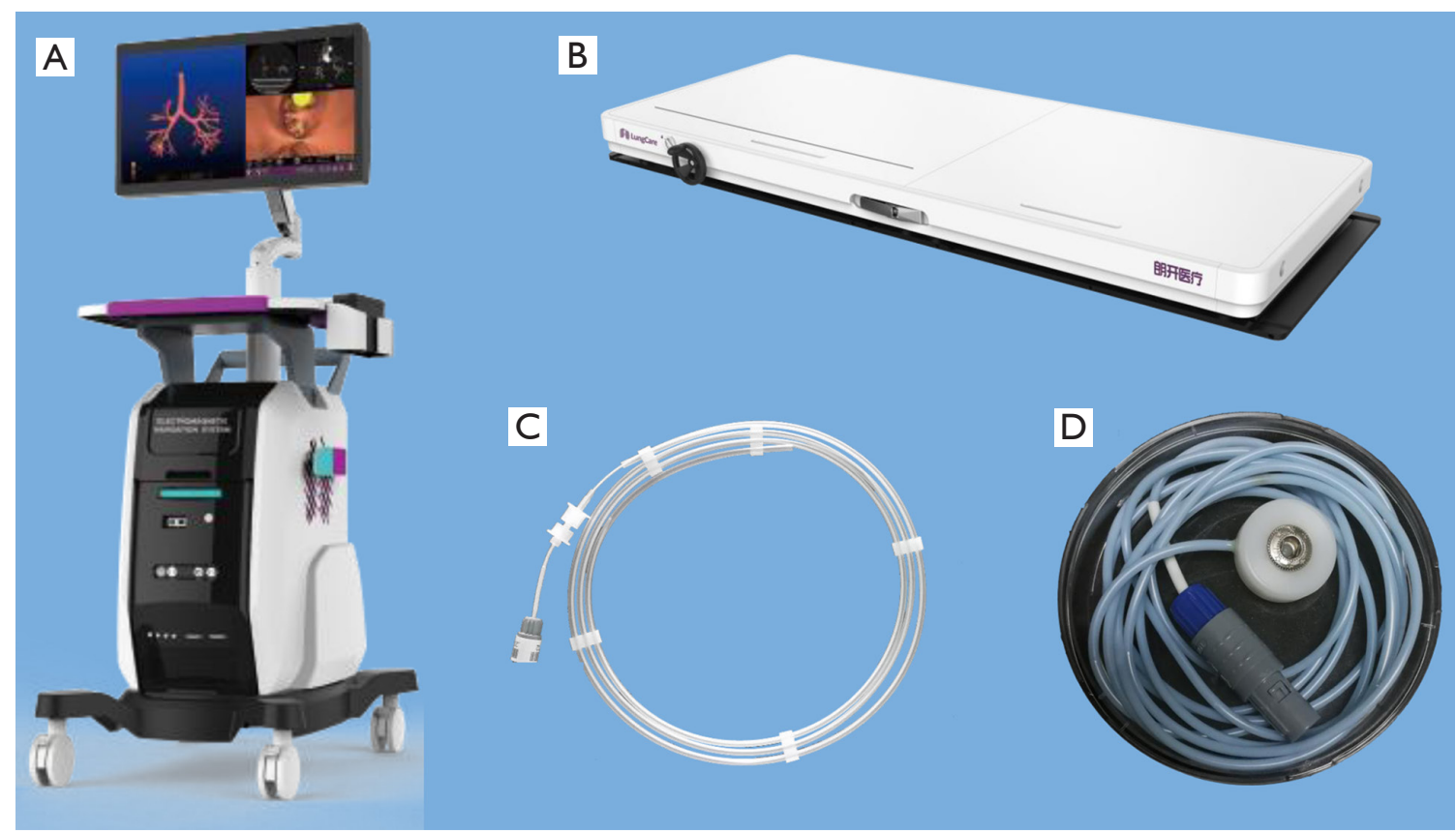

Figure 1 Components of electromagnetic navigation bronchoscopy system. The electromagnetic navigation bronchoscopy system consists of (A) a workstation with computer software, (B) electromagnetic location board, (C) locatable wire, and (D) position detection wire.

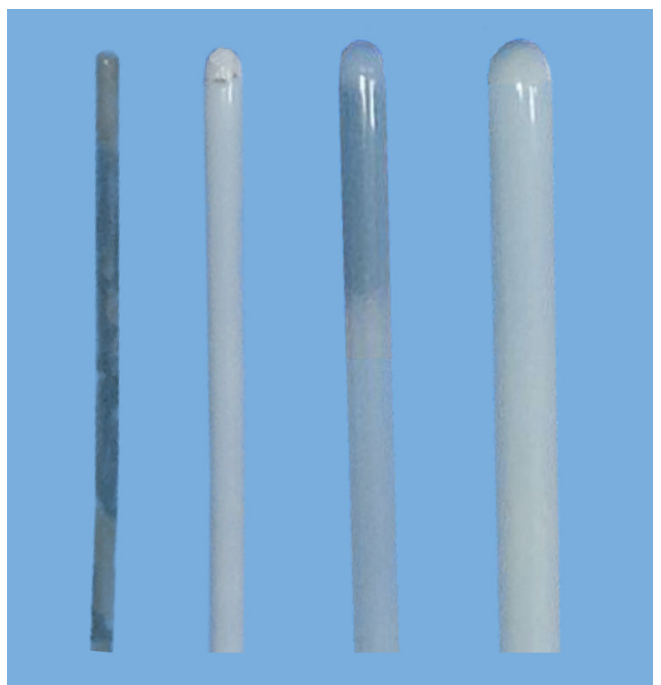

Figure 2 Locatable wires. There are four types of locatable wires with outer diameters of $0.75,1.15,1.45$ and $1.95 \mathrm{~mm}$ (from left to right).

bronchial tree showing the position of the tip of the locatable wire during real-time navigation.

The electromagnetic location board has a size of $1,800 \mathrm{~mm} \times 570 \mathrm{~mm} \times 89 \mathrm{~mm}$ and is placed underneath the back of the patient, from where it emits electromagnetic waves.

The tip of the locatable wire contains a sensor probe, and its position can be tracked within the electromagnetic field and displayed on the monitor. There are four types of locatable wires with different outer diameters $(0.75,1.15$, 1.45 , and $1.95 \mathrm{~mm}$ ) (Figure 2), which can be combined with the puncture needle and guide sheaths (GSs) and bronchoscopes of different sizes (Figure 3).

The position detection wire is used to collect the respiratory amplitude information and provides continuous intraoperative matching transferred to the workstation. There are three position detection wires that are connected to three electrodes attached to the chest on one end and the signal collector for transfer to the workstation on the other end.

\section{$G S$}

It is recommended to use two different kinds of GSs, for example, a thin GS (SG-200C; Olympus) and a thick GS (SG-201C; Olympus) with an outer diameter of 1.95 and $2.55 \mathrm{~mm}$, respectively, combined with different locatable wires and bronchoscope sizes. 


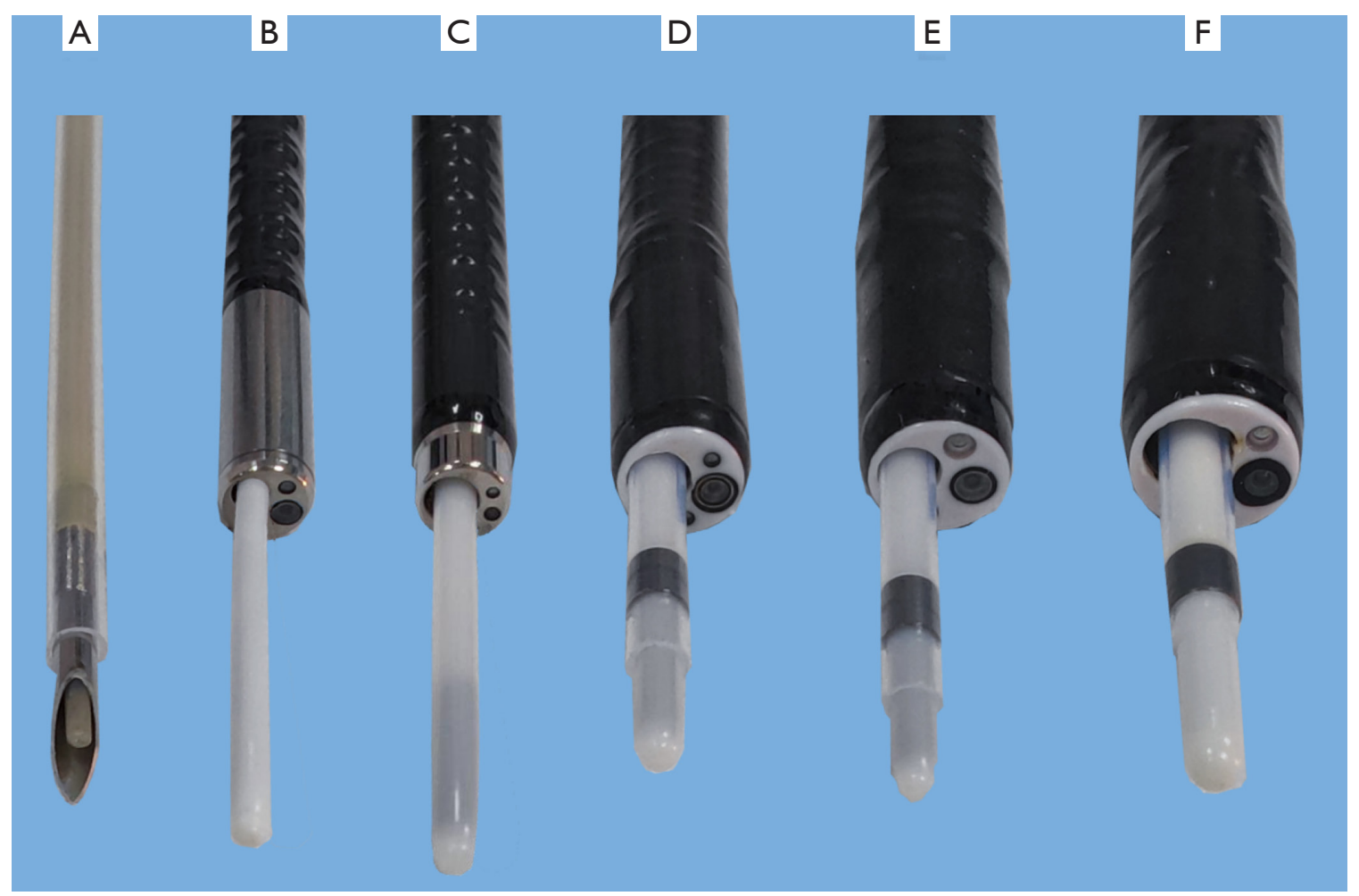

Figure 3 The usages of different types of locatable wires in compatible with puncture needle, guide sheaths and bronchoscopes. (A) A $0.75-\mathrm{mm}$ locatable wire in compatible with a puncture needle, (B) BF-XP290 (outer diameter 2.9 mm, working channel $1.2 \mathrm{~mm}$ ) in compatible with a $1.15-\mathrm{mm}$ locatable wire, (C) BF-MP290F (outer diameter $3.0 \mathrm{~mm}$, working channel $1.7 \mathrm{~mm}$ ) in compatible with a 1.45 -mm locatable wire, (D) BF-P290 (outer diameter $4.2 \mathrm{~mm}$, working channel $2.0 \mathrm{~mm}$ ) in compatible with a 1.95 -mm guide sheath and a 1.45 -mm locatable wire, (E) BF-Q290 (outer diameter $4.8 \mathrm{~mm}$, working channel $2.0 \mathrm{~mm}$ ) in compatible with a 1.95 -mm guide sheath and a 1.45-mm locatable wire, (F) BF-1TQ290 (outer diameter $5.9 \mathrm{~mm}$, working channel $3.0 \mathrm{~mm}$ ) in compatible with a 2.55 -mm guide sheath and a $1.95-\mathrm{mm}$ locatable wire.

\section{Radial endobronchial ultrasound (R-EBUS)}

Two types of R-EBUS probes are recommended for the combined use with ENB, including UM-S20-20R and UM-S20-17S (Olympus) with an outer diameter of 1.7 and $1.4 \mathrm{~mm}$, respectively.

\section{Sampling tools}

The sampling tools include biopsy forceps, cytology brush, puncture needle, and cryoprobe.

\section{Accessory equipment}

Accessory equipment commonly used is a C-arm with or without the function of $3 \mathrm{D}$ imaging. However, the $\mathrm{C}$-arm is not always necessary. Conventional biopsy or brushing can be performed without $\mathrm{C}$-arm guidance, but needle aspiration or cryo-biopsy is recommended to be performed under fluoroscopy to ensure accuracy and safety in the face of the relatively high risk of pneumothorax and bleeding.

\section{Standardized procedures}

\section{Preoperative preparation}

\section{Patients}

Patients should be informed of the purpose, costeffectiveness, benefits, and potential risk of ENB and provide written informed consent before the procedure. The preoperative examinations are the same as that for conventional bronchoscopy. During the procedure, the patient's electrocardiogram, blood pressure, pulse, and oxygen saturation have to be monitored. Any metal objects 
and mobile communication equipment carried by patients should be removed before the procedure so as not to affect navigation accuracy.

\section{Anesthesia}

ENB can be performed either under local anesthesia with or without moderate sedation or general anesthesia. A laryngeal mask is recommended for general anesthesia. The preoperative assessment will enable the anesthetist to select the most appropriate anesthesia and airway management methods according to an individual patient's situation and the planned sampling approach.

\section{Establishing preoperative venous access}

Preoperative venous access should be established before the procedure to facilitate the intraoperative use of medication, and the intravenous line should be kept during the recovery phase afterward.

\section{Chest CT}

Patients should have a thin-slice contrast-enhanced chest CT scan with slices of $0.5-1 \mathrm{~mm}$ thickness at $0.5-1 \mathrm{~mm}$ interval and an image resolution of $512 \times 512$ pixels in the $\mathrm{DICOM}^{\circledR}$ international standard for medical images and related information format.

\section{Planning}

The chest-CT data are transferred to the workstation to reconstruct the axial, coronal, and sagittal views of the lung as well as the virtual bronchial tree. The bronchi are segmented by the software, but manual segmentation is possible if the bronchioli cannot be segmented automatically. Target lesions are depicted according to their size and shape, which are shown on the three different views and the virtual bronchial tree. The software creates the optimal access route to the lesion automatically once the target lesion is located. The correction point can be set within the most distal bronchus the bronchoscope can reach to be able to correct potential deviations during the realtime navigation.

\section{Intraoperative workflows}

Specimens can be obtained with different bronchoscopy guidance methods. The optimal approach is selected based on the location and characteristics of the lesion. The commonly used methods are thin bronchoscopy combined with ENB-EBUS with a GS (ENB-EBUS-GS), or ultrathin bronchoscopy combined with ENB-EBUS alone. ENB-guided TBLB can be performed with or without fluoroscopy. The primary roles of fluoroscopy are to confirm that the lesion has been reached and to monitor the sampling procedure. The following describes the use of thin bronchoscopy with GS as well as ultrathin bronchoscopy without GS in details.

\section{ENB combined with thin bronchoscopy with or without fluoroscopy \\ Preparation of the GS}

The ultrasonic probe, locatable wire, cytology brush, and biopsy forceps are inserted into the GS, and their distal ends are brought into the appropriate position. Then, the proximal end is marked using the lock (Figure 4).

\section{Routine bronchoscopy}

In the case the procedure is performed with fluoroscopy, a positioning photo is taken using the $\mathrm{C}$-arm. White light bronchoscopy is routinely performed to examine the glottis, trachea, main carina, left and right main bronchus, and each lobe, segment, and subsegment bronchus to observe the lumina and, at the same time, remove secretions.

\section{Registration}

After concluding the bronchoscopy examination, the bronchoscope is kept at the carina while the GS-covered locatable wire is inserted. The bronchoscope is then advanced into the left and right main bronchus before being pulled back to the trachea to finish the registration. The software automatically establishes virtual bronchial tree images, which are then matched with the real images observed during bronchoscopy. The software displays the matching degree on the screen, and re-registration should be conducted if the matching degree is below $80 \%$. The operator should then follow the route created by the software to direct the bronchoscope into the target bronchus after waiting for three respiratory cycles as shown by the respiratory curve on the monitor to ensure a regular breathing pattern to reduce correction errors.

\section{Real-time navigation}

The ENB system provides multiple planar reconstruction images, including horizontal, sagittal, and coronal CT images, a virtual 3D bronchial tree, and virtual bronchoscopy images to track and observe the position of the tip of the locatable wire in relation to the target lesion from different angles in real time. The ENB system further depicts the exact distance of the tip of the locatable wire to the center of the PPL in real time. The navigation can be corrected through the preset correction point if it is off 


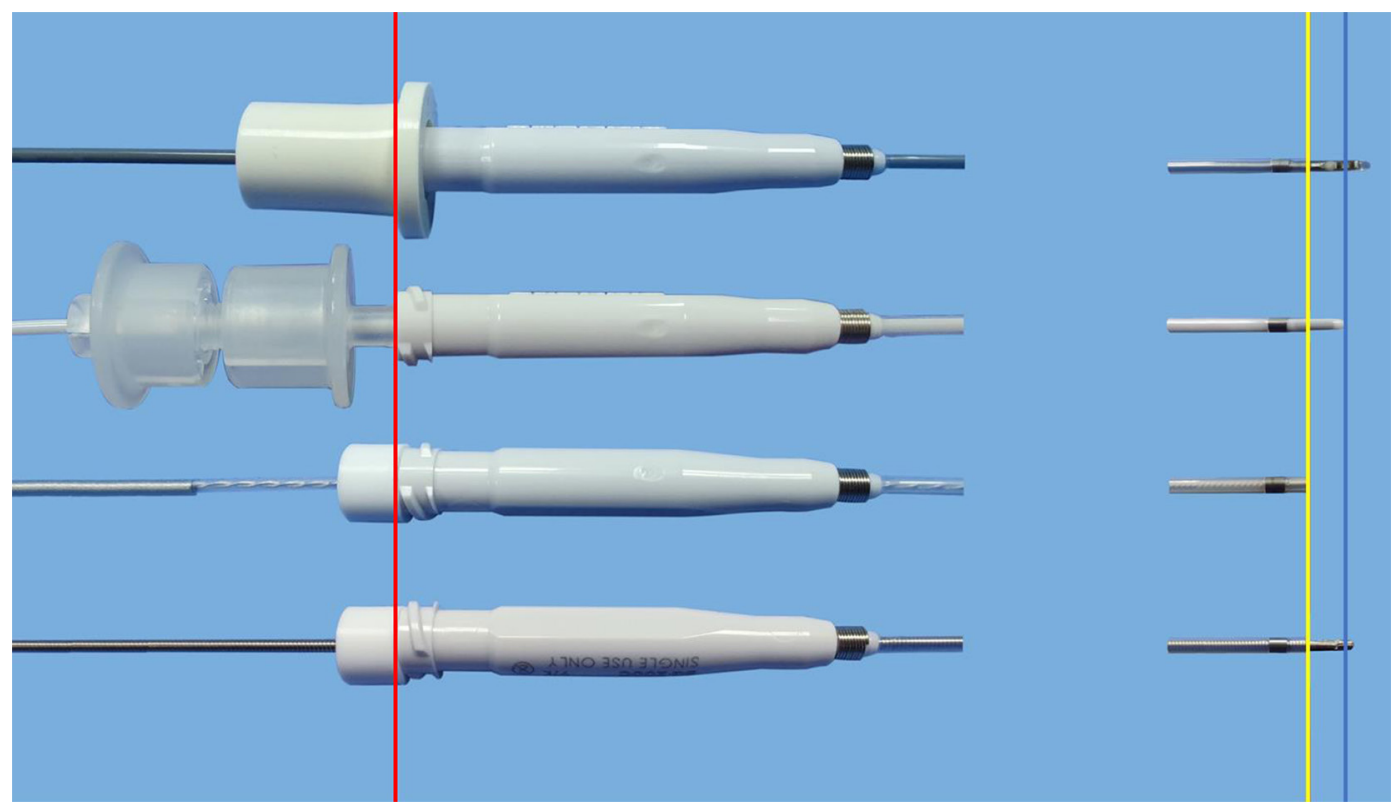

Figure 4 Preparation of guide sheath. This is a $1.45-\mathrm{mm}$ locatable wire and a radial endobronchial ultrasound (UM-S20-17S; Olympus) in compatible with a guide sheath kit (K-201; Olympus), used with standard or thin bronchoscope. The ultrasonic probe (outer diameter $1.4 \mathrm{~mm}$ ), locatable wire (outer diameter $1.45 \mathrm{~mm}$ ), cytology brush (outer diameter $1.4 \mathrm{~mm}$ ), and biopsy forceps (outer diameter $1.5 \mathrm{~mm}$ ) are inserted into the guide sheath (outer diameter $1.95 \mathrm{~mm}$ ), and their distal ends are brought into the appropriate position (blue line). The proximal end is marked using the lock (red line). The yellow line indicates the distal end of the guide sheath.

route during the real-time navigation because of CT-tobody divergence. If the bronchoscope cannot be advanced during the navigation, the operator needs to continue to advance the locatable wire toward the target following the navigational path. Whether the locatable wire reaches the lesion is determined by observing the distance between the tip of the locatable wire and the center of the PPL, CT images of different dimensions, the $3 \mathrm{D}$ virtual bronchial tree, and virtual bronchoscopy images.

\section{Obtaining R-EBUS images}

When the locatable wire reaches the target lesion, the operator removes it from the GS while keeping the GS in situ. The ultrasonic probe is inserted into the GS and switched on to confirm the probe has reached the lesion and whether it is located within, adjacent to, or outside according to the ultrasonic characteristics. The operator adjusts the position to obtain the typical ultrasonic image, measure the lesion size in B-mode, and obtain the image. If fluoroscopy is used, it is performed to confirm the probe is correctly positioned before measuring.

In the next step, the position of the GS should be marked when the ultrasound probe is just proximal to the lesion. Another image of the position of the ultrasound probe should be obtained at this time as a reference for the advancement of the sampling instrument to the lesion if fluoroscopy is used. The probe is then closed and removed from the GS, leaving the GS in place.

\section{Specimen collection}

The operator then inserts the cytology brush and biopsy forceps into the GS to obtain cytological and histological specimens, respectively. The previously taken fluoroscopic image of the probe position serves as a reference to adjust the position of the tools if fluoroscopy is used. Specimens are obtained in the following sequence: brushing, biopsy, brushing, and flushing. At least five visible specimens should be collected (16). The GS is left in situ for about one minute after sampling to prevent bleeding and withdrawn from the working channel thereafter. The GS is flushed with normal saline, and the flushing fluid is collected for cytology or microbiology examination. Rapid on-site evaluation (ROSE) can be performed to assess specimen eligibility and preliminary examination results. For lesions without the bronchus sign, a puncture needle can be used to obtain cytological samples.

\section{Removing the bronchoscope}

The bronchoscope is also removed from the airway if no 


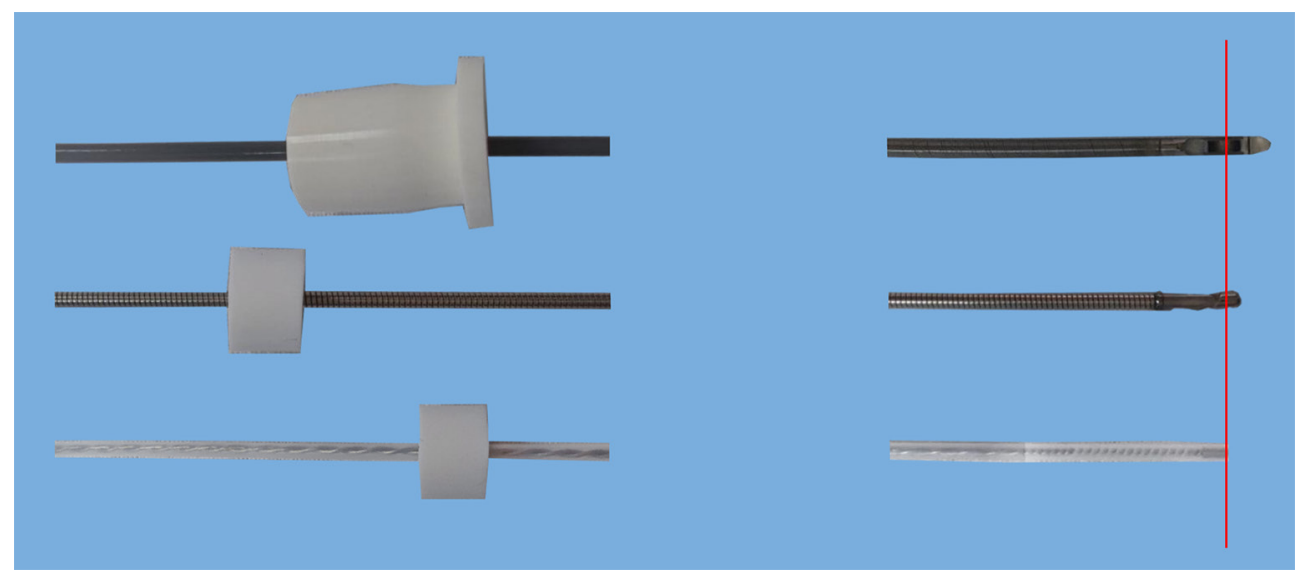

Figure 5 Preparation of sampling instruments without guide sheath. The operator adjusts the cytology brush (outer diameter 1.4 mm) and biopsy forceps (outer diameter $1.5 \mathrm{~mm}$ ) to the same level as the center of the ultrasonic transducer (red line) and marks the proximal end using the lock. This method often uses with ultrathin bronchoscope that is in compatible with a 1.4-mm radial endobronchial ultrasound.

bleeding is observed. Fluoroscopy can be used to exclude a pneumothorax before concluding the procedure.

The standardized procedure of ENB-EBUS-GS without fluoroscopy is demonstrated in Video 1.

\section{ENB combined with ultrathin bronchoscopy with or without fluoroscopy \\ Routine bronchoscopy, registration phase, and navigation phase}

Routine bronchoscopy and the registration and navigation phases are performed in the same way as described for thin bronchoscopy. Because ultrathin bronchoscopy cannot be combined with a GS, the locatable wire is inserted directly into the working channel of the bronchoscope (Figure 3C).

\section{Obtaining R-EBUS images}

Once the locatable wire reaches the target lesion, it is removed from the bronchoscope that is kept in situ. The ultrasonic probe is inserted into the bronchoscope and switched on to confirm its correct position. Lesion size is measured in B-mode, and the image is obtained as aforementioned.

The position of the ultrasonic probe is marked when it is just proximal to the lesion, and another fluoroscopic image of the position of the ultrasound probe is obtained. The probe is then closed and removed from the bronchoscope, which stays in place.

\section{Preparation of sampling instruments}

The operator adjusts the cytology brush and biopsy forceps to the same level as the center of the ultrasonic transducer and marks the proximal end using the lock (Figure 5).

\section{Specimen collection}

The cytology brush and biopsy forceps are introduced into the working channel of the bronchoscope to obtain cytological and histological specimens, respectively. The previously taken fluoroscopic image of the probe position serves as a reference to adjust the position of the tools if fluoroscopy is used. Specimens are obtained in the same sequence as described above: brushing, biopsy, brushing, and washing. Washing is performed within the target bronchus with normal saline, and the washing fluid is collected for cytology or microbiology examination.

\section{Removing the bronchoscope}

The bronchoscope can be removed from the airway if no bleeding is observed after sampling. Fluoroscopy can be used to exclude a pneumothorax before concluding the procedure.

\section{Management of common complications}

Patients should be assessed for their physical fitness before bronchoscopy and routinely monitored during the procedure to reduce the risk of complications (17). The largest prospective study of bronchoscopy outcomes so far reported a total complication rate of $1.08 \%$ and a mortality of $0.02 \%$ (18). The overall complication rate of R-EBUS in the diagnosis of PPLs was $2.8 \%$, whereas a pneumothorax occurred in $1.4 \%$, bleeding in $1.1 \%$, and pneumonia in $0.3 \%$ of patients (19). Hayama et al. reported an overall complication rate of EBUS-GS of $1.3 \%$, a pneumothorax rate of $0.8 \%$, and pulmonary infection rate of $0.5 \%(20)$. 
Common complications of ENB-guided TBLB are similar to R-EBUS-guided TBLB and include pneumothorax, hemorrhage, hypoxemia, and pulmonary infection. Previous meta-analyses reported that ENB-guided TBLB had an overall complication rate of $4.2-4.4 \%$, with the common complications of pneumothorax occurring in $2.0-3.1 \%$ and minor bleeding in $0.9-1.0 \%(3,4)$.

\section{Pneumothorax}

Previous studies reported that a pneumothorax occurs in between $1 \%$ and $6 \%$ of patients during TBLB, and $40-70 \%$ of these patients required intercostal drainage (18,21-24). The latest meta-analysis of R-EBUS in the diagnosis of PPL reported a pooled incidence rate of pneumothorax of $0.7 \%$ (25). The pneumothorax rate was related to patients' age and the number of TBLBs (26,27). Durakovic et al. reported a low $2 \%$ pneumothorax rate of R-EBUS without a GS and fluoroscopy in patients with PPLs (28).

Therefore, it is not necessary to routinely perform a chest $\mathrm{X}$-ray in patients after bronchoscopy $(26,29)$. However, if a patient is symptomatic and a pneumothorax is suspected, a chest X-ray should be performed as soon as possible to confirm or exclude the diagnosis. The management of pneumothorax in these patients follows the established guidelines (30). Observation is the treatment of choice for a small pneumothorax without significant breathlessness. A large pneumothorax and/or breathlessness require active intervention (needle aspiration or chest drain insertion).

\section{Hemorrbage}

Hemorrhage is a common complication in patients undergoing biopsy. TBLB has been reported to cause mildto-moderate bleeding in $0.8 \%$ (31). TBLB has a higher incidence of severe bleeding than endobronchial biopsy, but the overall risk is small, and the majority of cases resolve spontaneously or with endoscopic instillation of adrenaline or iced saline $(14,17,32)$. Treatment is based on the severity of bleeding and should follow the recommendations in the relevant guidelines (17).

\section{Hypoxemia}

In the majority of patients, hypoxemia is transient during bronchoscopy, and no specific intervention is required $(17,33)$. If deemed necessary, hypoxemia can be treated with oxygen supplementation via a nasal or pharyngeal catheter in a procedure performed under local anesthesia or mechanical ventilation in a procedure performed under general anesthesia.

\section{Pulmonary infection}

Post-operative fever is often transient and resolves spontaneously since it is most commonly caused by a noninfective acute inflammatory response. A bacterial infection is rare, with the pulmonary infection rate after R-EBUS combined with GS reported to be $0.5 \%$ (20). If a patient has persistent fever for more than 24 hours or an increased white blood cell count, microbiological examination and antibiotic treatment should be considered. Antibiotic prophylaxis before bronchoscopy does not prevent pulmonary infection (17).

\section{Key issues}

\section{Recommendations for the type of bronchoscope to use}

We recommend thin bronchoscopes combined with ENB and GS for the diagnosis of PPL. This ENB system can be performed in combination with thick bronchoscope, GS and large forceps. However, the GS used in combination with this novel ENB is not a pre-bent catheter and may not reach the peripheral lesion if it is used with a thick bronchoscope. Compared to thick bronchoscopes, thin bronchoscopes can reach bronchi that are further distal and confirm the lesion under direct vision and ENB navigation in distal bronchi that are invisible under the bronchoscope, thus shortening the examination time and reducing the manipulation of the bronchoscope, which is advantageous for patients with local anesthesia.

Ultrathin bronchoscopes with a 2.9-mm outer diameter and $1.2 \mathrm{~mm}$ working channel can be used only with small forceps without R-EBUS, providing very limited samples. The ultrathin bronchoscope with a $3.0-\mathrm{mm}$ outer diameter and $1.7 \mathrm{~mm}$ working channel can be used with R-EBUS and reach distal bronchi. But compared to a thin bronchoscope used with a $1.95-\mathrm{mm}$ GS, the ultrathin bronchoscope may not be placed close to the lesion, which might lead to a large deviation when the locatable wire removed from the working channel.

\section{Recommendations for R-EBUS}

Electromagnetic navigation can accurately guide the bronchoscope to the target lesion. However, the virtual bronchial tree constructed based on the preoperative CT 
scan is not entirely consistent with the actual bronchial tree because of the influence of respiratory movements and body position. Therefore, confirmation tools are needed to confirm the target lesion when the navigation shows that the target is reached. There are few studies on whether ENB combined with R-EBUS increases the diagnostic yield compared to ENB alone. Eberhardt et al. reported that the diagnostic yield of ENB combined with R-EBUS without fluoroscopy of $88 \%$ was greater than that of ENB alone $(59 \%)$ in a total of 118 patients (34). Furthermore, a later study of ENB showed that lesions that were located with R-EBUS had a higher diagnostic yield than when the location of lesions was not confirmed by R-EBUS (35). However, the NAVIGATE study reported a diagnostic yield of $70.6 \%$ for ENB combined with R-EBUS and $76.4 \%$ for ENB alone, which was not statistically significant based on the multivariate analysis (36). Of note, fluoroscopy was used in $91 \%$ of patients in the NAVIGATE study. Thus, the function of R-EBUS may be replaced by fluoroscopy, which might explain the absence of a statistically significant difference in the diagnostic yield between ENB alone and ENB combined with R-EBUS.

Still, previous studies have demonstrated a positive role of R-EBUS in diagnosing PPL $(19,25,37,38)$. R-EBUS is commonly used to confirm the location of the lesion and has unique advantages in TBLB. It indicates the relationship between the bronchus and the target lesion and defines the nature of the lesion based on the image characteristics without exposing patients to radiation. This is especially valuable in lesions with ground-glass opacity or those are obscured by the ribs, heart, and diaphragm and are invisible with fluoroscopy. Therefore, we recommend R-EBUS in ENB-guided TBLB for the diagnosis of PPL.

\section{Recommendations for fuoroscopy}

Conventional bronchoscopy to diagnose PPLs was commonly performed under fluoroscopy, with a sensitivity of $34 \%$ and $63 \%$ for peripheral lesions $<2$ and $>2 \mathrm{~cm}$ in diameter, respectively (12). With the emergence of guided bronchoscopy, the diagnostic yield has improved considerably. The pooled sensitivity of R-EBUS and ENB for the diagnosis of lung cancer is $72 \%$ and $71-82 \%$, respectively $(3,4,25,39,40)$. The role of fluoroscopy in TBLB is to guide the GS, R-EBUS, and sampling instruments to the target lesion, to confirm that the GS, R-EBUS, and sampling instruments reach the target lesion, to identify a displacement of the GS or sampling instruments, and to monitor the sampling procedure. However, this guidance and confirmation can be achieved by ENB and R-EBUS. In these circumstances, the primary role of fluoroscopy is to monitor the biopsy procedure. Studies reported that R-EBUS-guided TBLB without fluoroscopy was effective, especially in lesions $>2 \mathrm{~cm}$ in diameter $(41,42)$. The $A C C P$ guidelines indicate that ENB-guided TBLB can be performed with or without fluoroscopic guidance, and it has been found to be complementary to R-EBUS (12). Theoretically, TBLB can be performed without fluoroscopy whenever a bronchus leading to or adjacent to the PPL is confirmed on thin-slice CT. Therefore, fluoroscopy is not necessary for the diagnosis of PPL in ENB combined with R-EBUS-guided TBLB. However, we recommend fluoroscopy for lesions in some situations that are difficult to diagnose (such as small lesions and those susceptible to displacement of biopsy tools, and those susceptible to procedure-related complications) or that undergo needle aspiration or cryo-biopsy.

\section{Recommendations for the use of sampling tools}

Sampling tools are selected according to the characteristics and location of the PPL. The relatively small $1.5 \mathrm{~mm}$ forceps have been widely used during ultrathin bronchoscopy with a $1.7-\mathrm{mm}$ working channel and for bronchoscopy with EBUS-GS with a high diagnostic yield for PPL (15). However, standard biopsy forceps or ultrathin cryoprobe is recommended for lesions with ground-glass opacity. Ground-glass opacity lesions require large-volume specimens to be diagnosed. Standard biopsy forceps or ultrathin cryoprobes can provide a larger specimen compared with the small forceps, especially an ultrathin cryoprobe obtains a high diagnostic yield for the diagnosis of ground-glass opacity lesions. In PPLs without a bronchus leading or adjacent to them, a puncture needle is recommended because it is very challenging to obtain biopsy specimens with the forceps.

\section{Recommendations for the use of ROSE}

A meta-analysis indicated that ROSE could increase the sensitivity of R-EBUS in diagnosing PPL but included only a small number of studies (23). A prospective randomized controlled study demonstrated that ROSE might improve the diagnostic yield and shorten the operation time for R-EBUS in the diagnosis of PPLs. Biopsy tools cannot be confirmed by electromagnetic navigation in real time. 
Therefore, ROSE provides feedback on the specimens obtained during the procedure. Until further evidence of its value emerges, we recommend using ROSE if it is available to evaluate the acceptability of specimens.

\section{Summary of the recommendations}

(I) Thin bronchoscopes combined with the novel ENB and GS are recommended for the diagnosis of PPL.

(II) The usage of R-EBUS is recommended in ENBguided TBLB for the diagnosis of PPL.

(III) Fluoroscopy is not necessary for the diagnosis of PPL in ENB combined with R-EBUS-guided TBLB, but it is recommended for lesions that are difficult to diagnose and for needle aspiration or cryo-biopsy.

(IV) Sampling tools are selected according to the characteristics and location of the PPL.

(V) ROSE is recommended in ENB-guided TBLB for the diagnosis of PPL if it is available.

\section{Conclusions}

This consensus statement specifies the use of a novel ENB system with different types of locatable wires in combination with different bronchoscopes. The statement standardizes the procedures for the use of this novel ENB system in the diagnosis of PPL. This consensus statement will be continuously updated with the accumulation of more evidence in the use of the system, further development of the device and its components, and new functions.

\section{Acknowledgments}

Funding: This work was supported by the National Key R\&D Program of China (2017YFC0112700), Shanghai Municipal Health and Medical Talents Training Program (2018BR09), and Shanghai Municipal Education Commission-Gaofeng Clinical Medicine Grant Support (20181815).

\section{Footnote}

Conflicts of Interest: All authors have completed the ICMJE uniform disclosure form (available at http://dx.doi. org/10.21037/jtd-21-369). The authors have no conflicts of interest to declare.
Ethical Statement: The authors are accountable for all aspects of the work in ensuring that questions related to the accuracy or integrity of any part of the work are appropriately investigated and resolved.

Open Access Statement: This is an Open Access article distributed in accordance with the Creative Commons Attribution-NonCommercial-NoDerivs 4.0 International License (CC BY-NC-ND 4.0), which permits the noncommercial replication and distribution of the article with the strict proviso that no changes or edits are made and the original work is properly cited (including links to both the formal publication through the relevant DOI and the license). See: https://creativecommons.org/licenses/by-nc-nd/4.0/.

\section{References}

1. Schwarz Y, Greif J, Becker HD, et al. Real-time electromagnetic navigation bronchoscopy to peripheral lung lesions using overlaid CT images: the first human study. Chest 2006;129:988-94.

2. Leong S, Ju H, Marshall H, et al. Electromagnetic navigation bronchoscopy: A descriptive analysis. J Thorac Dis 2012;4:173-85.

3. Gex G, Pralong JA, Combescure C, et al. Diagnostic yield and safety of electromagnetic navigation bronchoscopy for lung nodules: a systematic review and meta-analysis. Respiration 2014;87:165-76.

4. Folch EE, Labarca G, Ospina-Delgado D, et al. Sensitivity and Safety of Electromagnetic Navigation Bronchoscopy for Lung Cancer Diagnosis: Systematic Review and Metaanalysis. Chest 2020;158:1753-69.

5. Wang Memoli JS, Nietert PJ, Silvestri GA. Metaanalysis of guided bronchoscopy for the evaluation of the pulmonary nodule. Chest 2012;142:385-93.

6. Dhillon SS, Harris K. Bronchoscopy for the diagnosis of peripheral lung lesions. J Thorac Dis 2017;9:S1047-S1058.

7. Sun J, Yi X, Zhang X, et al. Diagnostic Value of Electromagnetic Navigation Bronchoscopy With a Guide Sheath for Peripheral Pulmonary Lesions: a Randomized Controlled Trial. 16th World Conference on Lung Cancer 2015;2015 Sep 6-9; Denver, Colorado.

8. Zheng X, Cao L, Zhang Y, et al. A Novel Electromagnetic Navigation Bronchoscopy System Combined with Endobronchial Ultrasound for the Diagnosis of Peripheral Pulmonary Lesions: A Prospective Multicenter 
Randomized Controlled Clinical Trial. 2020 The World Congress of Bronchology and Interventional Pulmonology 2020;2020 Nov. 19-22; Shanghai, China.

9. Gould MK, Donington J, Lynch WR, et al. Evaluation of individuals with pulmonary nodules: when is it lung cancer? Diagnosis and management of lung cancer, 3rd ed: American College of Chest Physicians evidence-based clinical practice guidelines. Chest 2013;143:e93S-e120S.

10. Jiang S, Xie F, Mao X, et al. The value of navigation bronchoscopy in the diagnosis of peripheral pulmonary lesions: A meta-analysis. Thorac Cancer 2020;11:1191-201.

11. Chao TY, Lie CH, Chung YH, et al. Differentiating peripheral pulmonary lesions based on images of endobronchial ultrasonography. Chest 2006;130:1191-7.

12. Rivera MP, Mehta AC, Wahidi MM. Establishing the diagnosis of lung cancer: Diagnosis and management of lung cancer, 3rd ed: American College of Chest Physicians evidence-based clinical practice guidelines. Chest 2013;143:e142S-e165S.

13. Network Comprehensive Cancer Network. NCCN Clinical Practice Guidelines in Oncology. Non-Small Cell Lung Cancer. Available online: https://www.nccn.org/ professionals/physician_gls/default.aspx\#site. Accessed 28 February 2021.

14. Interventional pulmonology group of the Chinese Thoracic Society, Chinese Medical Association. Guideline for diagnostic flexible bronchoscopy in adults (2019). Zhonghua Jie He He Hu Xi Za Zhi 2019;42:573-90.

15. Oki M, Saka H. Diagnostic value of ultrathin bronchoscopy in peripheral pulmonary lesions: a narrative review. J Thorac Dis 2020;12:7675-82.

16. Yamada N, Yamazaki K, Kurimoto N, et al. Factors related to diagnostic yield of transbronchial biopsy using endobronchial ultrasonography with a guide sheath in small peripheral pulmonary lesions. Chest 2007;132:603-8.

17. Du Rand IA, Blaikley J, Booton R, et al. British Thoracic Society guideline for diagnostic flexible bronchoscopy in adults: accredited by NICE. Thorax 2013;68 Suppl 1:i1-i44.

18. Facciolongo N, Patelli M, Gasparini S, et al. Incidence of complications in bronchoscopy. Multicentre prospective study of 20,986 bronchoscopies. Monaldi Arch Chest Dis 2009;71:8-14.

19. Ali MS, Trick W, Mba BI, et al. Radial endobronchial ultrasound for the diagnosis of peripheral pulmonary lesions: A systematic review and meta-analysis. Respirology 2017;22:443-53

20. Hayama M, Izumo T, Matsumoto Y, et al. Complications with Endobronchial Ultrasound with a Guide Sheath for the Diagnosis of Peripheral Pulmonary Lesions. Respiration 2015;90:129-35.

21. Colt HG, Matsuo T. Hospital charges attributable to bronchoscopy-related complications in outpatients. Respiration 2001;68:67-72.

22. Pue CA, Pacht ER. Complications of Fiberoptic Bronchoscopy at a University Hospital. Chest 1995;107:430-2.

23. Milman N, Faurschou P, Munch EP, et al. Transbronchial lung biopsy through the fibre optic bronchoscope. Results and complications in 452 examinations. Respir Med 1994;88:749-53.

24. Ahmad M, Livingston DR, Golish JA, et al. The safety of outpatient transbronchial biopsy. Chest 1986;90:403-5.

25. Sainz Zuñiga PV, Vakil E, Molina S, et al. Sensitivity of Radial Endobronchial Ultrasound-Guided Bronchoscopy for Lung Cancer in Patients With Peripheral Pulmonary Lesions: An Updated Meta-analysis. Chest 2020;157:994-1011.

26. Izbicki G, Shitrit D, Yarmolovsky A, et al. Is routine chest radiography after transbronchial biopsy necessary?: A prospective study of 350 cases. Chest 2006;129:1561-4.

27. Hehn BT, Haponik E, Rubin HR, et al. The relationship between age and process of care and patient tolerance of bronchoscopy. J Am Geriatr Soc 2003;51:917-22.

28. Durakovic A, Andersen H, Christiansen A, et al. Retrospective analysis of radial EBUS outcome for the diagnosis of peripheral pulmonary lesion: sensitivity and complications. Eur Clin Respir J 2015;2:28947.

29. Milam MG, Evins AE, Sahn SA. Immediate chest roentgenography following fiberoptic bronchoscopy. Chest 1989;96:477-9.

30. MacDuff A, Arnold A, Harvey J, et al. Management of spontaneous pneumothorax: British Thoracic Society Pleural Disease Guideline 2010. Thorax 2010;65 Suppl 2:ii18-31.

31. Cordasco EM Jr, Mehta AC, Ahmad M. Bronchoscopically induced bleeding. A summary of nine years' Cleveland clinic experience and review of the literature. Chest 1991;100:1141-7.

32. Bjørtuft O, Brosstad F, Boe J. Bronchoscopy with transbronchial biopsies: measurement of bleeding volume and evaluation of the predictive value of coagulation tests. Eur Respir J 1998;12:1025-7.

33. Jones AM, O'Driscoll R. Do all patients require supplemental oxygen during flexible bronchoscopy? Chest 2001;119:1906-9.

34. Eberhardt R, Anantham D, Ernst A, et al. Multimodality 
bronchoscopic diagnosis of peripheral lung lesions: a randomized controlled trial. Am J Respir Crit Care Med 2007;176:36-41.

35. Eberhardt R, Morgan RK, Ernst A, et al. Comparison of suction catheter versus forceps biopsy for sampling of solitary pulmonary nodules guided by electromagnetic navigational bronchoscopy. Respiration 2010;79:54-60.

36. Folch EE, Pritchett MA, Nead MA, et al. Electromagnetic Navigation Bronchoscopy for Peripheral Pulmonary Lesions: One-Year Results of the Prospective, Multicenter NAVIGATE Study. J Thorac Oncol 2019;14:445-58.

37. Paone G, Nicastri E, Lucantoni G, et al. Endobronchial ultrasound-driven biopsy in the diagnosis of peripheral lung lesions. Chest 2005;128:3551-7.

38. Steinfort DP, Khor YH, Manser RL, et al. Radial probe endobronchial ultrasound for the diagnosis of peripheral lung cancer: systematic review and meta-analysis. Eur

Cite this article as: Xie F, Yang H, Huang R, Zheng X, Cao L, Liu J, Qu S, Zhang Y, Wu S, Zhang J, Ke M, Sun J; on behalf of the Interventional \& Minimally Invasive Respiratory Committee of the China Medical Education Association and Expert Assistance Group of the Domestic Electromagnetic Navigation Bronchoscopy Technology. Chinese expert consensus on technical specifications of electromagnetic navigation bronchoscopy in diagnosing peripheral pulmonary lesions. J Thorac Dis 2021;13(4):2087-2098. doi: 10.21037/jtd-21369
Respir J 2011;37:902-10.

39. Qian K, Krimsky WS, Sarkar SA, et al. Efficiency of Electromagnetic Navigation Bronchoscopy and Virtual Bronchoscopic Navigation. Ann Thorac Surg 2020;109:1731-40.

40. Zhang W, Chen S, Dong X, et al. Meta-analysis of the diagnostic yield and safety of electromagnetic navigation bronchoscopy for lung nodules. J Thorac Dis 2015;7:799-809.

41. Yoshikawa M, Sukoh N, Yamazaki K, et al. Diagnostic value of endobronchial ultrasonography with a guide sheath for peripheral pulmonary lesions without X-ray fluoroscopy. Chest 2007;131:1788-93.

42. Herth FJF, Eberhardt R, Becker HD, et al. Endobronchial ultrasound-guided transbronchial lung biopsy in fluoroscopically invisible solitary pulmonary nodules: a prospective trial. Chest 2006;129:147-50. 